\title{
Similarity Assessment by Multivariate Statistics Method Based on Distance Between Biosimilar and Originator
}

Jian Xu

State Key Laboratory of Bioreactor Engineering, Shanghai Collaborative Innovation Center for Biomanufacturing Technology

\section{Zhihui Shao}

CAS Key Laboratory of Synthetic Biology, CAS Center for Excellence in Molecular Plant Sciences, Institute of Plant Physiology and Ecology

\section{Xiaoxiong Han}

Process Development Department

\section{Yingfeng Huang}

R\&D Laboratories

\section{Xun Zou}

Shanghai Sanjin Bioscience and Technology

\section{Yaling Shen ( $\nabla$ ylshen@ecust.edu.cn )}

State Key Laboratory of Bioreactor Engineering, Shanghai Collaborative Innovation Center for Biomanufacturing Technology

\section{Research}

Keywords: biosimilar, similarity, glycosylation pattern, multivariate statistics method, cluster analysis

Posted Date: December 30th, 2020

DOl: https://doi.org/10.21203/rs.3.rs-135641/v1

License: (c) (i) This work is licensed under a Creative Commons Attribution 4.0 International License. Read Full License

Version of Record: A version of this preprint was published at Bioresources and Bioprocessing on March 29th, 2021. See the published version at https://doi.org/10.1186/s40643-021-00378-2. 


\section{Biosimilar and Originator}

$4{ }^{a}$ State Key Laboratory of Bioreactor Engineering, Shanghai Collaborative Innovation Center

5 for Biomanufacturing Technology, East China University of Science and Technology,

6 Shanghai, People's Republic of China

$7{ }^{b}$ CAS Key Laboratory of Synthetic Biology, CAS Center for Excellence in Molecular Plant

8 Sciences, Institute of Plant Physiology and Ecology, Chinese Academy of Sciences, Shanghai,

9 China;

$10{ }^{c}$ Process Development Department, Dragon Sail Pharmaceutical, Shanghai, China

$11{ }^{d}$ R\&D Laboratories, Dragonboat Biopharmacetical, Shanghai, China

12 e Shanghai Sanjin Bioscience and Technology, Shanghai, China

$13 *$ Corresponding authors

14 Yaling Shen (ylshen@ecust.edu.cn),

15 Tel: +86-2164253156, Fax: +86-2164250068,

16 Address: 130 Mei Long Road, Shanghai 200237, China.

\section{Abstract}

19 The development of biosimilar products or follow-on biologics has been flourishing in recent

20 years because of lower price than originators. In this study, a multivariate data analysis method based on JMP software was proposed to assess the glycosylation pattern similarity of antibody candidates from different conditions in optimization experiment with reference. A 
1 specific distance was generated by this method and indicated the glycoform similarity

2 between biosimilar and reference. This method can be applied to analyze the similarity of

3 other physicochemical and function characters between follow-on biologics and originators.

4 Then the design of experiments method can be realized to optimize culture conditions of cell

5 culture to attain similar antibody candidates.

6 Key words: biosimilar, similarity, glycosylation pattern, multivariate statistics method,

7 cluster analysis.

\section{1. Introduction}

9 During past two decades, biologic products (also termed "biopharmaceuticals") have been

10 developed intensively by companies for treating cancer and autoimmune diseases such as

11 rheumatoid arthritis. 6 of the top pharmaceutical products sales in 2019 were biologic

12 products (Table 1). So, there has been an increasing trend toward development of biosimilars

13 considering market interest and improving access of biologics. Biosimilar products can offer

14 lower price so as to optimize efficiencies across healthcare systems. However,

15 biopharmaceuticals typically involve expression of the gene using living cell followed by

16 purification and formulation to acquire stable drug product. They are large, complex and

17 heterogeneous compared to small molecular produces. It is impossible to manufacture

18 identical copies of biologic products (WHO 2016). To define biosimilar, different regulatory

19 body has different criteria. EMA define a biotherapeutic product that is similar in terms of

20 quality, safety, and efficacy to an already licensed reference product (reference medicinal

21 product) in the EMA (EMA 2015). While, FDA define a biological product that is highly

22 similar to a US-licensed reference product notwithstanding minor differences in clinically 
1 inactive components, and for which there are no clinically meaningful differences between

2 the biological product and the reference product in terms of safety, purity, and potency of the

3 product (FDA 2015b). WHO define a biotherapeutic product that is similar in terms of quality,

4 safety, and efficacy to an already licensed reference product (WHO 2016). In broad terms, a

5 biosimilar is highly similar to a reference product in terms of structure and function. And the

6 high similarities of physicochemical and function characters are main aim in pre-clinical

7 development phase.

8 The structural and functional elements of therapeutic antibody include primary structure,

9 purity, charge heterogeneity, glycosylation and other post-translational modifications, as well

10 as target and receptor bindings activity and bioactivity features (Kirchhoff et al. 2017). A

11 variety of analytic techniques are developed to demonstrate these elements. However, many

12 analytic results are multivariate data such as charge heterogeneity, glycosylation and size

13 heterogeneity. It is hard to use simple method to assess the similarity between biosimilar and

14 originator (or reference) based on these multivariate outcomes.

15 Identification of the glycosylation pattern is a key consideration during the development of 16 mono-clonal antibody $(\mathrm{mAb})$ biosimilars, since the glycan chains in the Fc region can 17 substantially alter protein activity and the PK profile, and in some case, antigenicity

18 (Kirchhoff et al. 2017). Because many cell culture conditions such as nutrient availability, pH,

19 dissolved oxygen (DO), ammonia, cell viability, growth phase, temperature will affect

20 glycosylation, the culture process parameters should be well monitored and controlled in

21 manufacture phase (Patrick et al. 2019). Chemical supplements such as metal ion and

22 substrate for glycan chain synthesis have been described in the literature as effective 
1 glycosylation modulators during up-stream process development (Crowell et al. 2006,

2 Gramer et al. 2011). To find the optimal addition amount, design of experiment (DOE) is

3 used intensively during screening experiment. But a specific parameter indicating the

4 similarity should be defined as response before modelling and ANOVA analysis. An effective

5 method that can output a specific parameter to assess the similarity of glycan profile is

6 needed.

7 In this work, a multivariate data analysis method was first applied to generate a specific index

8 that can represent the similarity of glycan profiles from different antibody candidates. By this

9 method, the similar antibodies were clustered by the glycan distribution and identified the

10 most similar antibody easily. And the effect of supplements could be qualified statistically.

11 2. Materials and methods

$12 \quad 2.1$ Cell line and reagents

13 The cell line expressing recombinant IgG1 was derived from CHO DG44. The basal media is

14 CD DF1 (Shanghai BasalMedia Technologies Co., LTD., Shanghai, China) supplemented

15 with $6 \mathrm{mM}$ Glutamine (Sigma, Shanghai, China). Feed media is Efficient Feed ${ }^{\mathrm{TM}} \mathrm{C}+$

16 (Thermofisher, Shanghai, China) and add additives as the design. The additives,

17 N-Acetyl-D-glucosamine (GlcNAc) and $\mathrm{MnCl}_{2}$ were purchased from Sigma-Aldrich

18 Shanghai Trading Co., Ltd (Shanghai, China).

192.2 Cell culture conditions and process design

20 The cells were cultured in shaker flasks (Corning, New York, USA) with vented cap at 5\%

$21 \mathrm{CO}_{2}, 70 \%$ humidity and $37^{\circ} \mathrm{C}$ (shift to $35^{\circ} \mathrm{C}$ from day 4 to harvest) condition. The volume of

22 beginning culture was $30 \mathrm{~mL}$ with $120 \mathrm{rpm}$ of shaking rate. Inoculum density was adjusted to 
11 million cells/mL equally. GlcNAc and $\mathrm{MnCl}_{2}$ were supplemented into culture by two ways:

2 adding in feed media, or adding separately into basal media on day 4. The supplemented

3 amount was designed as the table 2. The first 14 runs and second 14 runs were duplicated,

4 and the last 2 runs were control runs without supplemental. The feed was started from day 3

5 and the feed volume was calculated by the following equation.

6

$$
V_{\text {feed }}=V_{\text {current }} \times Q_{\text {feed }} \times \frac{\left(V C D_{1}+V C D_{2}\right)}{2 \times G l_{\text {feed }} \times 1000}
$$

7 In which, $\mathrm{V}_{\text {feed }}(\mathrm{mL})$ is the feed volume of current day; $\mathrm{V}_{\text {current }}(\mathrm{mL})$ is the culture volume

8 before feeding; $\mathrm{Q}_{\text {feed }}(75)$ is the feed rate factor; $\mathrm{VCD}_{1}\left(10^{6}\right.$ cell $\left./ \mathrm{mL}\right)$ is the viable cell density

9 of current day; $\mathrm{VCD}_{2}\left(10^{6}\right.$ cell/ $\left./ \mathrm{mL}\right)$ is calculated viable cell density of next day; and Glceed

$10(\mathrm{~g} / \mathrm{L})$ is the glucose concentration in feed media.

11 Sampling was conducted every day from day 3. The residual glucose was analyzed by

12 Glucose Test Kit (Beihai, China). Additional glucose was calculated by the following

13 equation and added to maintain the concentration of glucose at $3 \mathrm{~g} / \mathrm{L}$ when viable cell density

14 less than $1.0 \times 10^{7}$ cell $/ \mathrm{mL}$, and shift to $4 \mathrm{~g} / \mathrm{L}$ when viable cell density higher than $1.0 \times 10^{7}$

$15 \mathrm{cell} / \mathrm{mL}$.

$V_{\text {Glc }}=\left[\left(G l c_{\text {target }}-G l c_{\text {test }}\right) \times V+\left(G l c_{\text {target }}-G l c_{\text {test }}\right) \times V_{\text {feed }}\right] / G l c_{\text {solution }}$.

17 In which, VGlc (mL) is the addition volume of glucose solution; Glcfeed $(\mathrm{g} / \mathrm{L})$ is the glucose

18 concentration in feed media; Glctest $(\mathrm{g} / \mathrm{L})$ is the glucose concentration in culture; Glctarget

$19(\mathrm{~g} / \mathrm{L})$ is the target glucose concentration after feeding; and Glcsolution $(\mathrm{g} / \mathrm{L})$ is the

20 concentration of glucose solution.

21 After 11 days culture, the culture was harvested by centrifugation to remove cell debris and 
1 purified by Protein A resin.

$2 \quad 2.3$ Glycan Analysis

3 2.3.1 Enzymolysis of glycan chains from antibody

4 Firstly, replaced the buffer of the antibody sample with 50mmol/L $\mathrm{NH}_{4} \mathrm{HCO}_{3}(\mathrm{pH} 8.0)$. Then

$5500 \mu \mathrm{g}$ antibody was hydrolyzed with 2000 U PNGase F (New England Biolabs, Beijing,

6 China) at $37^{\circ} \mathrm{C}$ for $24 \mathrm{~h}$. Pre-cooled ethanol was added to the final concentration of $75 \%(\mathrm{v} / \mathrm{v}$ ).

7 The mixture was blended and stand for $0.5 \mathrm{~h}$ at $-20^{\circ} \mathrm{C}$. After centrifugation at $13000 \mathrm{rpm}$ for

815 min, the supernatant was vacuum dried.

$9 \quad$ 2.3.2 Fluorescence labeling of glycan chains

10 Add 5 mg 8-aminopyrene-1,3,6-trisulfonic acid trisodium salt (APTS, AB Sciex Pte. Ltd.,

11 Framingham, USA) into $0.5 \mathrm{~mL}$ aqueous solution containing $15 \%$ acetic acid and vortex.

12 Then add $15 \mu \mathrm{L}$ APTS solution and $5 \mu \mathrm{L}$ tetrahydrofuran solution containing $1 \mathrm{~mol} / \mathrm{L}$ sodium

13 cyanoborohydride to the vacuum-dried glycan chains. After fluorescent labeling at $55^{\circ} \mathrm{C}$ for

$142 \mathrm{~h}, 400 \mu \mathrm{L}$ ultrapure water was added and analyzed by PA800 Plus capillary electrophoresis

15 apparatus (AB Sciex Pte. Ltd., Framingham, USA).

\section{$16 \quad$ 2.3.3 Analysis by capillary electrophoresis apparatus}

17 Beckman N-Cho coated capillary with total length of $60.5 \mathrm{~cm}$ and effective length of $50 \mathrm{~cm}$ and inner diameter of $50 \mu \mathrm{m}$ and electrophoresis buffer was purchased from Beckman

19 Coulter Life Sciences (Indianapolis, USA). The capillary temperature was $20^{\circ} \mathrm{C}$. Sample was injected at $2.0 \mathrm{psi}$ for $10 \mathrm{~s}$. Then the sample was separated at $30 \mathrm{kV}$ for $20 \mathrm{~min}$. Fluorescence detection was implemented at excitation and emission wavelengths of 488 and $520 \mathrm{~nm}$, respectively. 
2 The peak area percentages from glycan analysis are used for assigning the similarity in this

3 case. Seven peaks are identified and coded numbers by isoform. In this way all the data is

4 normalized for each sample. A symmetrical matrix of the data is built and a Hierarchical

5 Cluster Analysis is performed with JMP software (SAS Institute Inc.). A dendrogram can be

6 generated and save the distance matrix to another data table.

\section{3. Results}

\section{$8 \quad 3.1$ Glycan Analysis}

9 The glycan peak distribution of 30 samples from different shake flask culture are shown in

10 Fig. 1. All the samples' glycan shows similar patten that peak 3 is the highest portion,

11 however the percentages of each peak are different. At a glance, the supplements of GlcNAc

12 and $\mathrm{MnCl}_{2}$ should affect the glycan distribution of antibodies. But it is difficult to test the

13 effect by statistic method, because a specific number to assign the similarity is lack to

14 indicate the variance of these peaks.

153.2 Cluster analysis

16 Perform the cluster analysis tool in JMP as described in method, a hierarchical clustering tree

17 is shown in Fig. 2. The single samples are the leaves and the similar samples are clustered on

18 one branch. We have set the duplicate runs same color, so it is shown that almost all duplicate

19 samples are in third or fourth branch from main trunk. It is very clear to see that SF-7, 8, 13,

$2014,21,22,27,28$ are located in same third branch with reference, which illustrates these samples' glycan distribution is very close to that of reference.

\subsection{Distance}


1 The distance is to show the similarity between samples and reference. They can be generated

2 by run saving the distance matrix option in menu. The distances are plot to run label is shown

3 in Fig. 3. Here is very clear to see that the antibody from SF-14 and 28 are the nearest to

4 reference antibody in aspect of glycan distribution, while SF-6 and 20 are the farthest. So as

5 to confirm the real peak distribution similarity, only SF-14, SF-20 and reference are shown in

6 Fig. 4. It is easy to find that SF-14 is similar to reference and the difference between SF-20

7 and reference is significant.

\section{$8 \quad 3.4$ Effect Significance Analysis}

9 Use the "Fit model" in JMP, set the Distance as the response and put GlcNAc, $\mathrm{MnCl}_{2}$ and

10 adding them in basal or feed media in model effect window, run the fit program. The ANOVA

11 results are shown in Table 3. In the effect test, GlcNAc and add in basal or feed media are

12 significant factor and $\mathrm{MnCl}_{2}$ is not. By this way, the significance of factors can be identified

13 in a statistic method.

\section{4. Discussion}

15 In developing a biosimilar drug, a stepwise approach is needed beginning with chemistry

16 manufacturing controls (CMC) and bioanalytical characterization (Burchiel et al. 2019). In

17 CMC development phase, because the structure of antibody is very complex, the quality attributes will be characterized by multiple analysis method such as glycan isoform, capillary

19 isoelectric focusing (cIEF), cation-exchange chromatography (CEX) and peptide map. We

20 know that these methods output multivariance results rather than a single data. Clustering is a

21 ubiquitous data analysis tool to divide complex data into groups of similar items (Andreas et

22 al. 2019). Therefore, it can be utilized to reveal the similarity of these multivariance test 
1 results effectively. Seung-Ho Kang et al (2013) proposed a three-arm parallel design to assess

2 biosimilarity between a biosimilar product and an innovator biological product based on

3 relative distance of means observed from the test and reference products. In the proposed

4 design, if the relative distance is less than a prespecified margin, they claim that the two

5 products are claimed to be biosimilar. This method's merit is to provide a specific standard to

6 access similarity. Beyond this method, we do not find other strategy to access biosimilar

7 quality by a statistic pathway in publish.

8 The clustering program in JMP can output hierarchical tree, making the cluster results

9 visualized and easy to find the similar group with reference. This can be utilized to control

10 the batch quality in manufacture phase. If the third branch is set as the similar margin, the

11 leaves of different batches data can be judged as qualified batches. Once a batch's quality is

12 clustered to other third branch, deviation investigation can be triggered and to recall or

13 destroy the batch based on risk assessment. The distance between samples and reference can

14 be used as response value in optimization experiment. By this way, quality by design (QbD)

15 strategy is feasible to character the process effect and optimize or define operation space of

16 critical process parameter (CPP) by DOE. In future, lots of batches would be clustered, a

17 distance can be set as margin to decide qualified batch too.

\section{5. Conclusion}

19 In this paper, a multivariate statistics method is proposed to access the similarity of antibodies to reference from different conditions in optimization experiment. The multivariance test

21 results can be grouped by this method and a specific distance can be generated. The distance

22 value indicates the similarity between biosimilar and reference. In this study, the glycoform 
1 with shortest distance shows highest similar to the reference glycoform. When a specific

2 value can indicate the similarity, the DOE method can be realized to evaluate the effect of

3 factors and optimize culture conditions.

4

\section{Abbreviations}

6 WHO: World health orgnization; EMA: European medicines agency; FDA: U.S. Food and 7 drug administration; mAb: mono antibody: PK: Pharmacokinetics; DO: Dissolved oxygen;

8 DOE: Design of experiment; ANOVA: Analysis of Variance; GlcNAc:

9 N-Acetyl-D-glucosamine; CMC: Chemical, manufacture and control; cIEF: Capillary

10 isoelectric focusing; CEX: Cation-exchange chromatography; QbD: Quality by design; CPP:

11 Critical process parameter;

\section{Declarations}

14 Ethics approval and consent to participate

15 Not applicable

16 Consent for publication

17 Not applicable

18 Availability of data and materials

19 Not applicable

20 Competing interest

21 The authors confirm that there are no conflicts of interest.

22 Funding 
$1 \quad$ Not applicable

\section{Authors' contributions}

3 Jian $\mathrm{Xu}$ wrote the main manuscript text. Zhihui Shao and Xiaoxiong Han analyzed the data

4 by statistic tool. Yingfeng Huang, Xun Zou and Yaling Shen insturcted the experiment.

\section{Acknowledgements}

6 The authors wish to acknowledge the Quality Analytical Science group in Dragonboat

7 biopharmaceutical Co., for testing the glycosylation.

\section{$8 \quad$ Authors' information (optional)}

9 Not Applicable

\section{References}

11 Andreas A, Margareta A, Naomi C. B (2019) To cluster, or not to cluster: An analysis of

12 clusterability methods. Pattern Recognition. 88:13-26.

13 https://doi.org/10.1016/j.patcog.2018.10.026

14 Burchiel S W, Aspbury R, Munday J (2019) The search for biosimilars and biobetters. Drug

15 Discovery Today. 24(5):1087-1091. https://doi.org/10.1016/j.drudis.2019.03.016

16 Crowell C K, Grampp G E, Rogers G N, Miller J, Scheinman R I (2006) Amino acid and

17 manganese supplementation modulates the glycosylation state of erythropoietin in a $\mathrm{CHO}$

18 culture system. Biotechnology \& Bioengineering. 96(3):538-549.

19 https://doi.org/10.1002/bit.21141

20 EMA. (2015) Guideline on similar biological medicinal products containing

21 biotechnology-derived proteins as active substance: Non-clinical and clinical issues.

22 Available online at: 
1 http://www.ema.europa.eu/docs/en_GB/document_library/Scientific_guideline/2015/01/W

$2 \quad$ C5001802 19.pdf.

3 FDA. (2015b) Guidance for industry: Scientific considerations in demonstrating biosimilarity

4 to a reference product. Available online at:

5 http://www.fda.gov/downloads/Drugs/GuidanceComplianceRegulatoryInformation/Guidan

$6 \quad$ ces/UCM291128.pdf

7 Gramer M J, Eckblad J J, Donahue R, Brown J, Shultz C, Vickerman K, Priem P, Bremer E,

8 Gerritsen J, Berkel P (2011) Modulation of antibody galactosylation through feeding of

9 uridine, manganese chloride, and galactose. Biotechnology \& Bioengineering.

10 108(7):1591-1602. https://doi.org/10.1002/bit.23075

11 Kirchhoff C F, Wang X Z M, Conlon H D, Anderson S, Ryan A M, Bose A (2017) Biosimilars:

12 Key regulatory considerations and similarity assessment tools. Biotechnology and

13 Bioengineering. 114(12):2696-2705. https://doi.org/10.1002/bit.26438

14 Patrick H, Khattak S F, Li Z J (2009) Optimal and consistent protein glycosylation in

15 mammalian cell culture. Glycobiology. 19(9):936-949.

16 https://doi.org/10.1093/glycob/cwp079

17 Kang S H, Chow S C (2013) Statistical assessment of biosimilarity based on relative distance

18 between follow-on biologics. Stats in Medicine. 32(3):382-392.

19 https://doi.org/10.1002/sim.5582

20 WHO. (2016) Guidelines on evaluation of monoclonal antibodies as similar biotherapeutic

21 products (SBPs). Available online at:

22 https://www.who.int/biologicals/biotherapeutics/WHO_TRS_1004_web_Annex_2.pdf. 
2 Table 1 The top 10 pharmaceutical products sold in 2019*.

\begin{tabular}{ccccc}
\hline Rank & Medicine & Sales (Billion \$) & Company & Type \\
\hline $\mathbf{1}$ & Humira & 19.17 & Abbvie & Antibody \\
\hline $\mathbf{2}$ & Eliquis & 12.15 & BMS/Pfizer & Small Moledular \\
\hline $\mathbf{3}$ & Keytruda & 11.08 & MSD & Antibody \\
\hline $\mathbf{4}$ & Revlimid & 7.171 & Celgene & Small Moledular \\
\hline $\mathbf{5}$ & Imbruvica & 8.09 & J\&J/Abbvie & Small Moledular \\
\hline $\mathbf{6}$ & Opdivo & 8.00 & BMS & Antibody \\
\hline $\mathbf{7}$ & Eylea & 7.44 & Bayer/Regeneron & Fusion Protein \\
\hline $\mathbf{8}$ & Avastin & 7.12 & Roche & Antibody \\
\hline $\mathbf{9}$ & Xarelto & 6.93 & Bayer & Small Moledular \\
\hline $\mathbf{1 0}$ & Enbrel & 6.92 & Amgen/pfizer & Fusion Protein \\
\hline
\end{tabular}

3 Note: * Data from the annual statement of the company.

4

5

6

7

8

9

10

11

12

13

14 
2 Table 2 The design of experiment for investigating glycan profile adjustment.

\begin{tabular}{|c|c|c|c|}
\hline Run & GlcNAc (mM) & $\mathrm{MnCl} 2(\mu \mathrm{M})$ & Add in Basal or Feed Media \\
\hline SF-1 & 120 & 30 & Feed \\
\hline SF-2 & 80 & 30 & Feed \\
\hline SF-3 & 40 & 30 & Feed \\
\hline SF-4 & 120 & 15 & Feed \\
\hline SF-5 & 80 & 15 & Feed \\
\hline SF-6 & 40 & 15 & Feed \\
\hline SF-7 & 120 & 0 & Feed \\
\hline SF-8 & 80 & 0 & Feed \\
\hline SF-9 & 40 & 0 & Feed \\
\hline SF-10 & 20 & 0 & Basal \\
\hline SF-11 & 10 & 0 & Basal \\
\hline SF-12 & 5 & 0 & Basal \\
\hline SF-13 & 10 & 30 & Basal \\
\hline SF-14 & 10 & 15 & Basal \\
\hline SF-15 & 120 & 30 & Feed \\
\hline SF-16 & 80 & 30 & Feed \\
\hline SF-17 & 40 & 30 & Feed \\
\hline SF-18 & 120 & 15 & Feed \\
\hline SF-19 & 80 & 15 & Feed \\
\hline SF-20 & 40 & 15 & Feed \\
\hline SF-21 & 120 & 0 & Feed \\
\hline SF-22 & 80 & 0 & Feed \\
\hline SF-23 & 40 & 0 & Feed \\
\hline SF-24 & 20 & 0 & Basal \\
\hline SF-25 & 10 & 0 & Basal \\
\hline SF-26 & 5 & 0 & Basal \\
\hline SF-27 & 10 & 30 & Basal \\
\hline SF-28 & 10 & 15 & Basal \\
\hline SF-29 & 0 & 0 & N/A \\
\hline SF-30 & 0 & 0 & N/A \\
\hline
\end{tabular}


2 Table 3 Analysis of four factors effecting on glycan similarity using distance value as the response.

\begin{tabular}{cccccc}
\hline Source & Nparm & DF & Sum of Squares & F Ratio & Prob $>\mathrm{F}$ \\
\hline GlcNAc & 1 & 1 & 173.1 & 27.2 & $<0.0001^{*}$ \\
$\mathrm{MnCl}_{2}$ & 1 & 1 & 17.6 & 2.8 & 0.1083 \\
$\begin{array}{c}\text { Add in Basal or Feed } \\
\text { Media }\end{array}$ & 2 & 2 & 474.2 & 37.3 & $<0.0001^{*}$ \\
\hline
\end{tabular}

3

4

5

6

7

8

9

10

11

12

13

14

15

16

17

18

19 


\section{Figure Captions}

3 Fig. 1 Glycan peak distribution of 30 samples for different shake flask culture.

4 Fig. 2 Hierarchical clustering tree analyzed by JMP software.

$5 \quad$ Fig. 3 Euclidean distance of 30 antibody candidates to reference.

6 Fig. 4 Glycan peak distribution similarity comparison of SF-14, SF-20 and reference.

7

8

9

10

11

12

13

14

15

16

17

18

19

20

21

22 


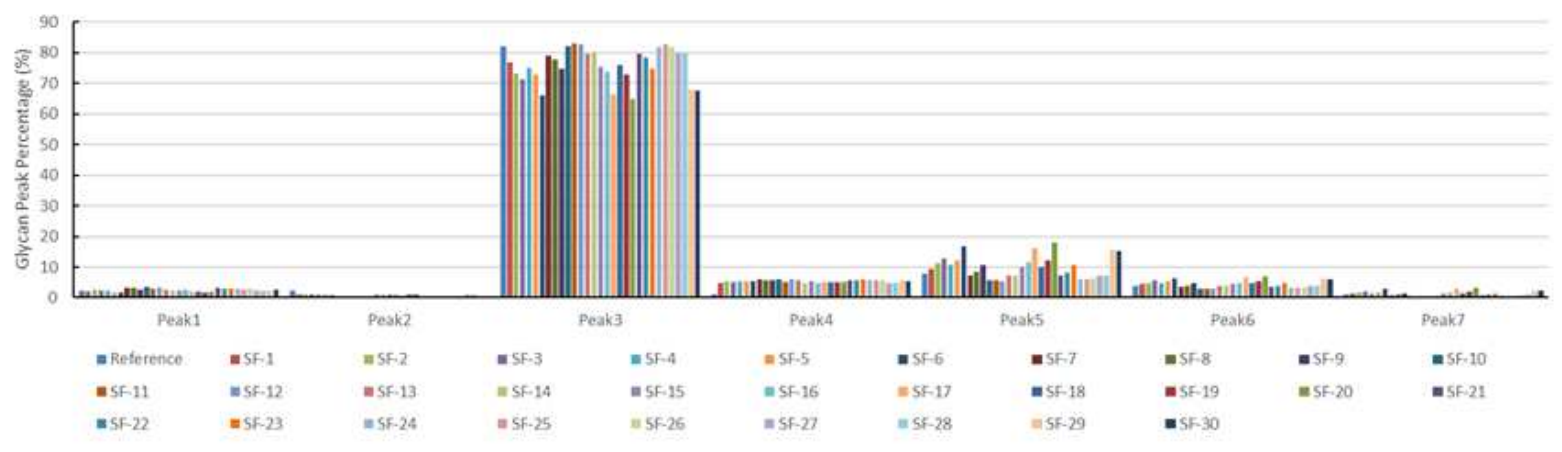

2

Fig. 1

3

4

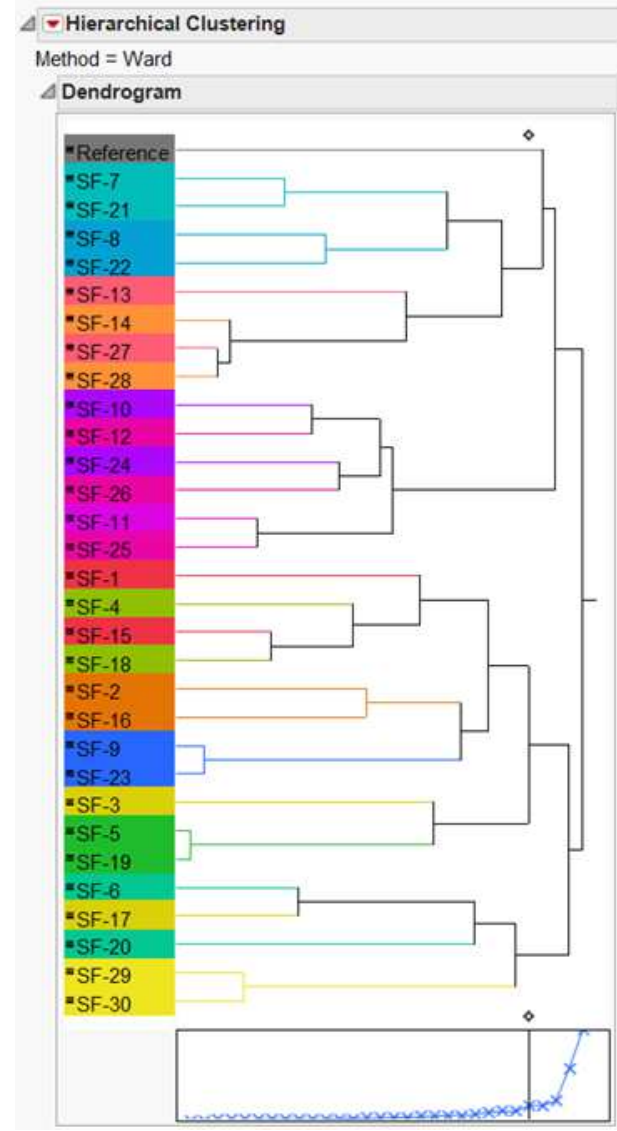

Fig. 2 


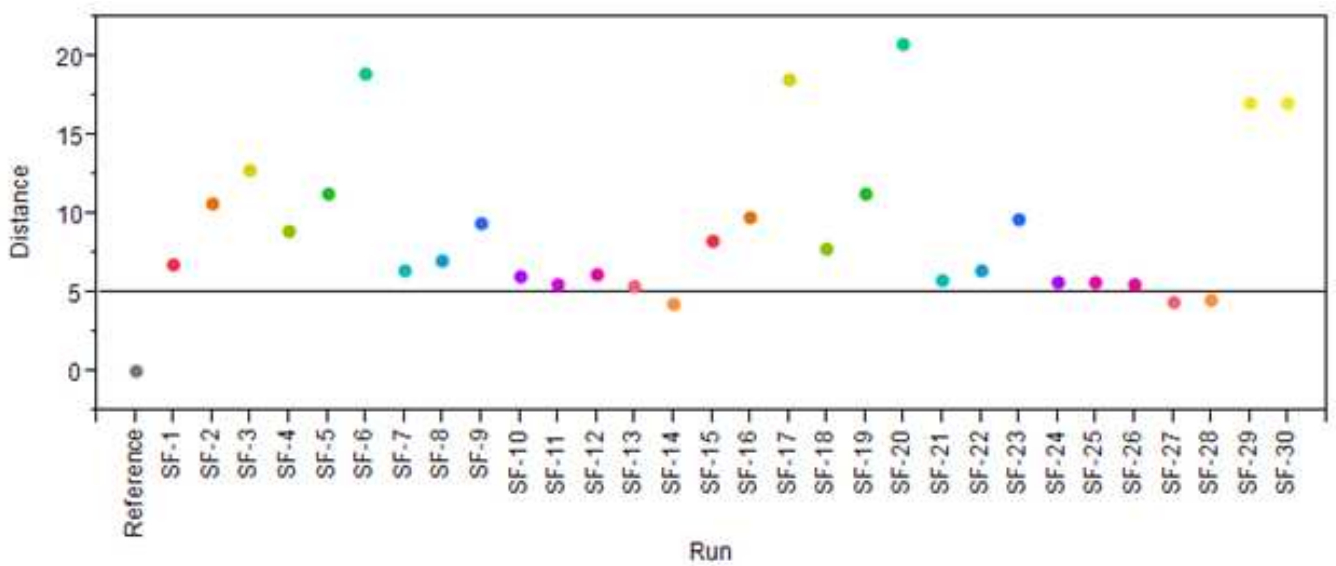

Fig. 3

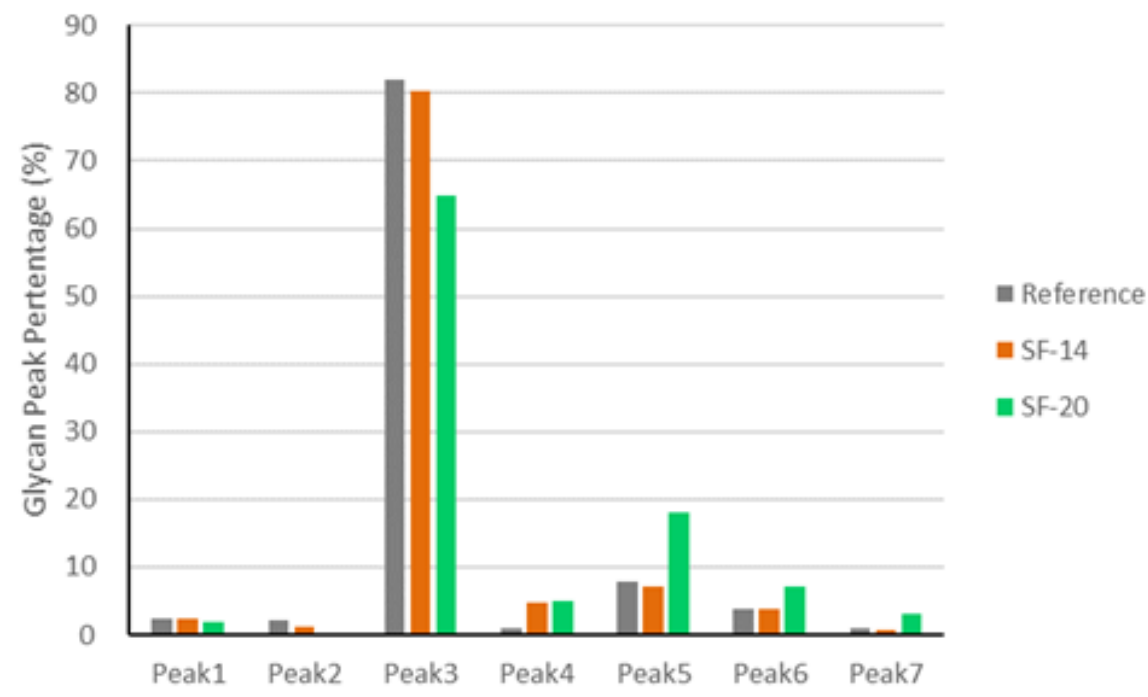

Fig. 4

6

7 
Figures

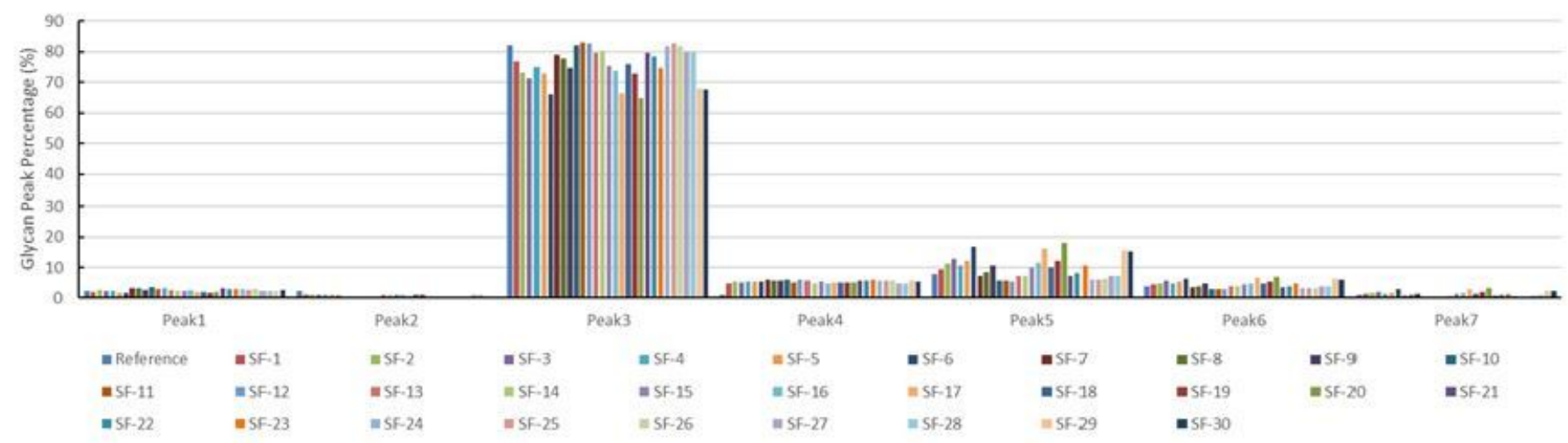

\section{Figure 1}

Glycan peak distribution of 30 samples for different shake flask culture 


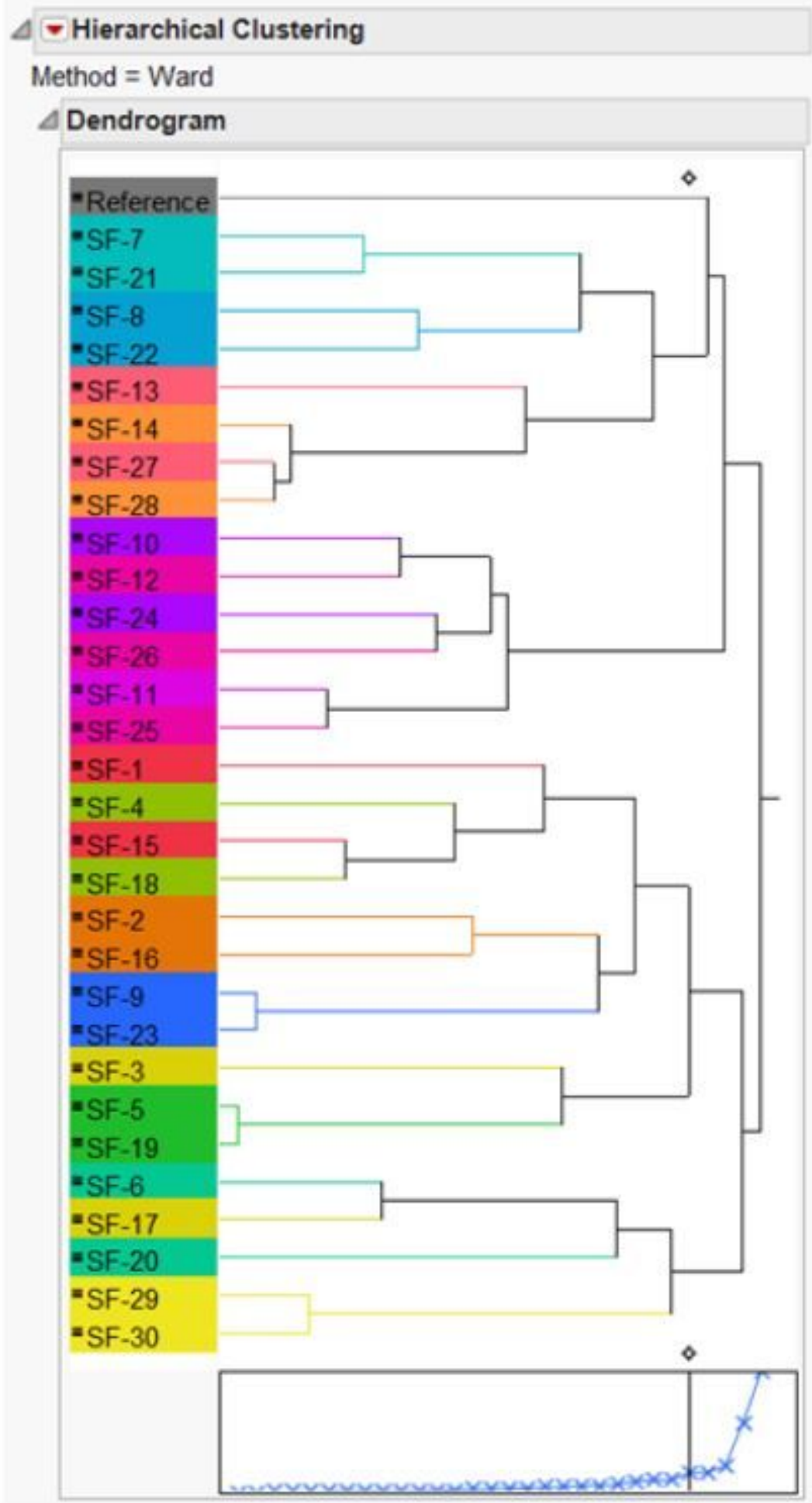

Figure 2

Hierarchical clustering tree analyzed by JMP software. 


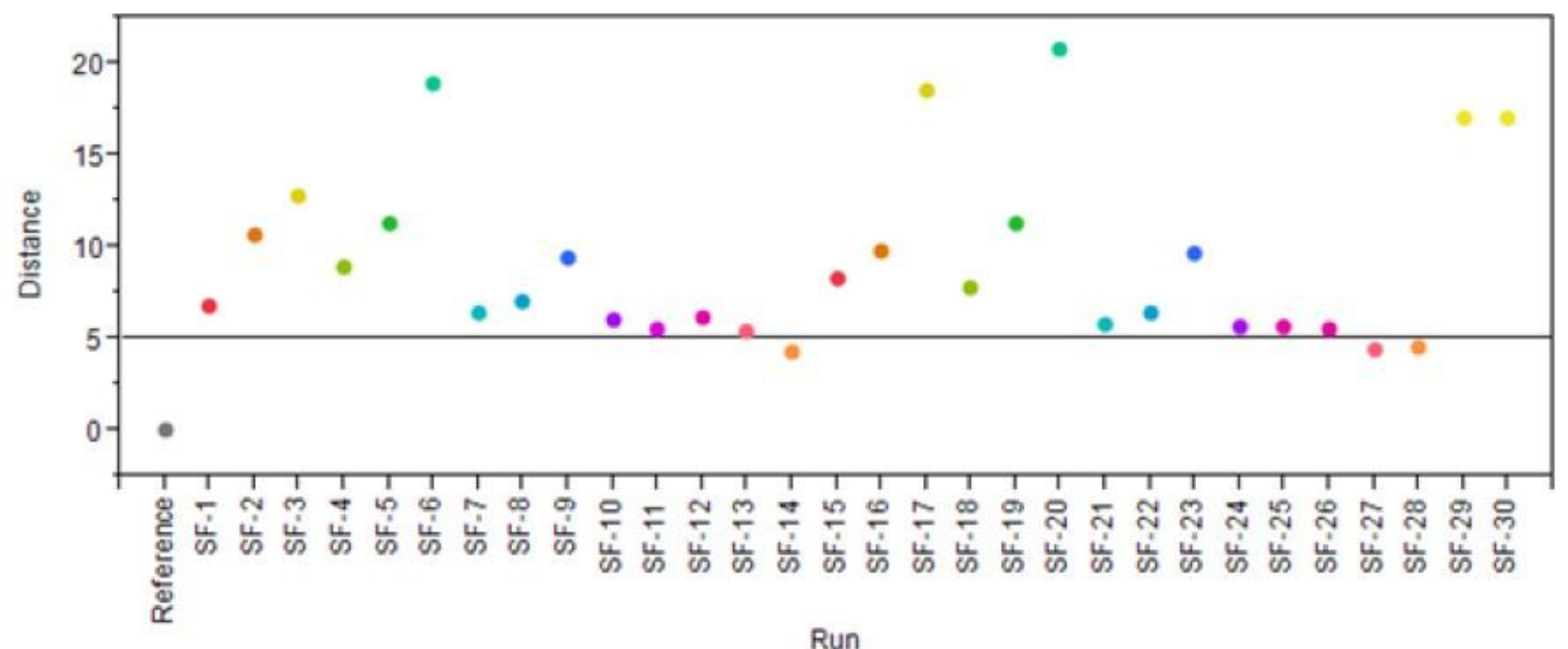

Figure 3

Euclidean $\mathrm{d}$ is tance of 30 an tibody candidates to reference.

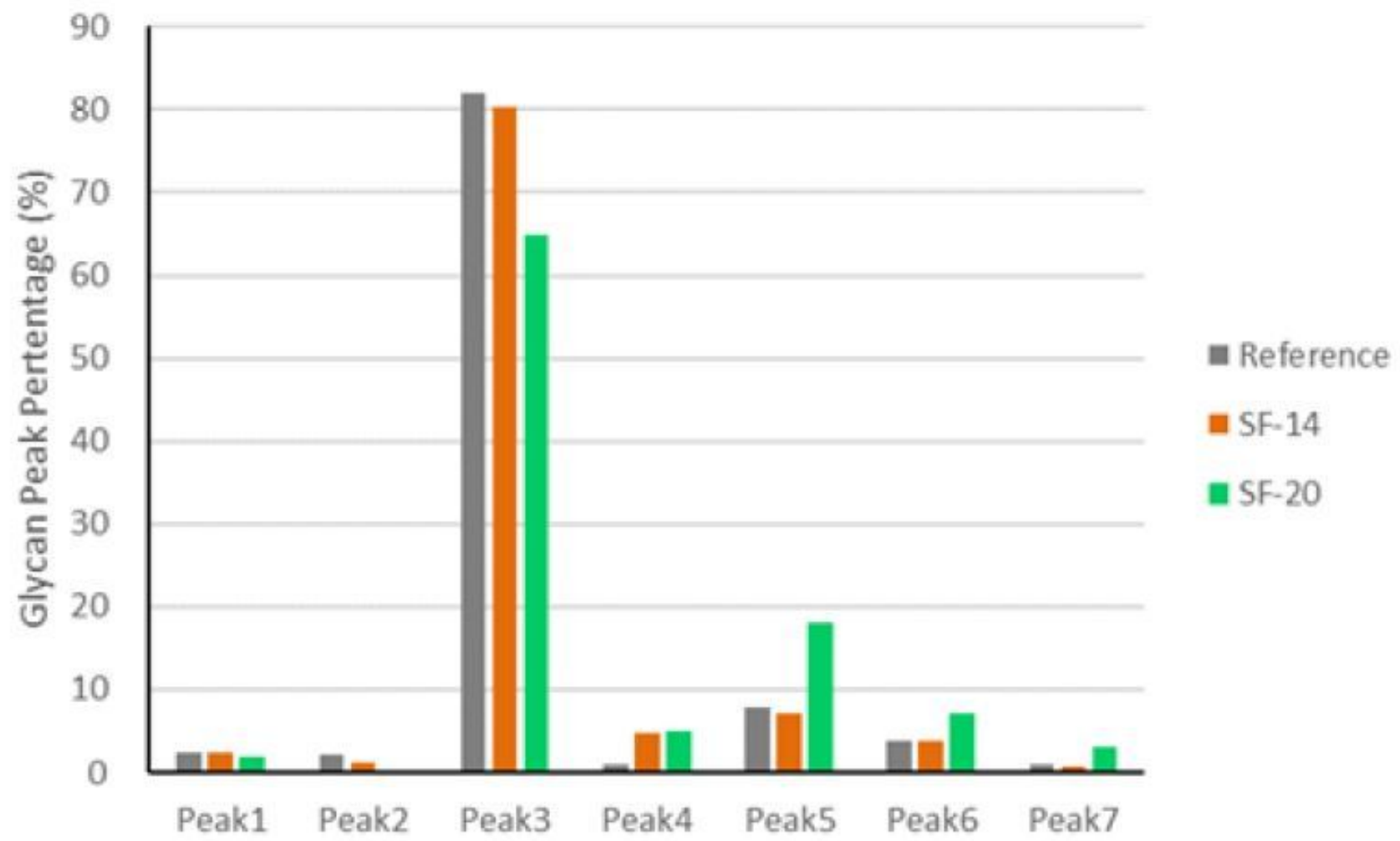

Figure 4

Glycan peak distribution similarity com parison of SF 14, SF 20 and reference 


\section{Supplementary Files}

This is a list of supplementary files associated with this preprint. Click to download.

- GraphicAbstract.png 